\title{
Experimental Research on Single Factor Ultrasonic Assisted Extraction of Polysaccharides from Gracilaria lemaneiformis
}

\author{
Yong-Guang $\mathrm{BI}^{1}$, Zhong WANG ${ }^{2}$, Hai-Lan $\mathrm{HUANG}^{1}$,Xue-Wei $\mathrm{CHEN}^{1}$,Xue-Mei \\ LIU $^{1}$, Hong $\mathrm{YU}^{1}$ and Shi-Ting DENG ${ }^{1}$ \\ ${ }^{1}$ School of Pharmacy, Guangdong Pharmaceutical University, Guangzhou 510006, Guangdong, \\ China
}

${ }^{2}$ School of Clinical Medicine, Guangdong Pharmaceutical University, Guangzhou 510006, Guangdong, China

E-mail:biyongguang2002@163.com

${ }^{*}$ Corresponding author

Keywords: Gracilaria lemaneiformis, ultrasonic, extraction, single factor, test.

\begin{abstract}
In this paper, the use of ultrasound-assisted extraction of polysaccharides from Gracilaria discussed ultrasonic power, ultrasonic time, four factors affect the extraction temperature and ratio of liquid to solid on the extraction rate, test results show that: with the increase of liquid ratio, the dragon shall dishes polysaccharide extraction rate is also rising, the impact of ultrasonic power on extraction of polysaccharide from Gracilaria increases after ultrasonic power and the first increase in a downward trend in the ultrasonic power between $280-320 \mathrm{~W}$ to achieve the best, with the increased ultrasonic time, the extraction rate of polysaccharide from Gracilaria presented firstly increased and then decreased. With the improvement of ultrasonic temperature, the extraction rate of polysaccharides from Gracilaria showing a decreasing trend after the first increase in about $55^{\circ} \mathrm{C}$ maximum extraction rate of $40.1 \%$, indicating that the asparagus dish suitable ultrasonic temperature at about $55^{\circ} \mathrm{C}$.
\end{abstract}

\section{Introduction}

Algae affected their own living environment, its chemical composition and terrestrial plants are quite different than terrestrial plants contain more abundant biologically active substances, medicinal ingredients, can be used as the new health food and medicinal resources ${ }^{[1]}$. In addition, a wide range of marine algae, the large number of resource-rich, showing originality and diversity of features. Biological studies have shown that a large number of results: Seaweed is not only enriched by some medicinal ingredients, but also by the reaction of natural active substances, thus pharmaceutical thematic research on algae and seaweed functional food increasing, extracted from algae organisms active substances with different health care function and effect of the world has become an active area of scientific research. The application of modern extraction, separation technology in extracting non-polar marine algae contain or weakly polar physiologically active substance, and the function and composition analysis, can provide the basis for the third generation of functional food research and development, scientific and technological strength for the contribution to the health of mankind.

Polysaccharides are a class of important organic compound organism, it is the material basis of life. Different types of polysaccharides, the exercise of different functions in the organism. In plant tissues, the main function of polysaccharides are: energy resources (storage polysaccharides), (an attribute finer structure of the special polysaccharide) structural strength (structural polysaccharide) and in the survival and distribution of competitive ecosystem ${ }^{[2]}$. After the 1960 s, people gradually found biologically active polysaccharide many ways, and most non-toxic, may be an ideal source of the drug. Such as laminarin anticoagulant effects of heparin and chondroitin sulfate to prevent hardening of the arteries, a variety of edible fungi polysaccharides with enhanced immune function and anti-cancer effects and the like.

Polysaccharide is composed of many monosaccharide molecules from past bond connecting 
natural polymers (polymer), the monosaccharide composition may be the same or different. In the same for the common, uniform called polysaccharides, such as starch, cellulose, glycogen and the like; polysaccharides with different monosaccharide called heteropolysaccharide, e.g., gum arabic are composed of galactose and arabinose[3-5]. In the biological world, it is a kind of polysaccharide widely present in animal cell membranes, cell walls of plants and microorganisms in biological macromolecules, is one of the four fundamental physical block of life, is an important component of all living organisms. Compared with other biomolecules such as lipids, nucleic acids, proteins, polysaccharides composed of more complex structure, but also diverse functions. Over the years polysaccharide has been considered a signal molecule in vivo, involved in immune recognition and signal transduction. Seaweed polysaccharide has a unique immune function, as medical and health care products has attracted more and more attention around the world, seaweed polysaccharide is gradually becoming a major source of biological polysaccharide.

Belongs to a class of polysaccharides from seaweed seaweed extract, has a wide range of application. Such as: agar, carrageenan, alginate industry has long been in use ${ }^{[6]}$. However, the function of seaweed polysaccharides as potential drugs and drug intermediates, only in recent years to be recognized that seaweed polysaccharides exhibit anticoagulant activity, antitumor activity and antiviral activity and so on. In this paper, ultrasonic assisted extraction of seaweed (asparagus dishes in the research object) polysaccharide to explore the impact of various factors on the extraction rate of polysaccharide from Gracilaria, the experimental study of this paper, in order to provide reference for the subsequent process optimization.

\section{Experimental Materials and Methods [7]}

\section{Determination of the Extraction Rate of Polysaccharide from Gracilaria}

Phenol - sulfuric acid method The content of polysaccharides, polysaccharide extraction yield (Y) is calculated as follows:

$$
Y=\frac{C \times D \times V \times 10^{-6}}{W} \times 100 \%
$$

Where: C- polysaccharide concentration corresponding to a standard curve, $\mu \mathrm{g} \cdot \mathrm{mL}-1$; Ddilution; V- extract volume, $\mathrm{mL}$; W- quality of raw material, g.

\section{Results and Discussion}

\section{Effect of Liquid Feed Ratio on the Extraction Rate of Polysaccharide from Gracilaria}

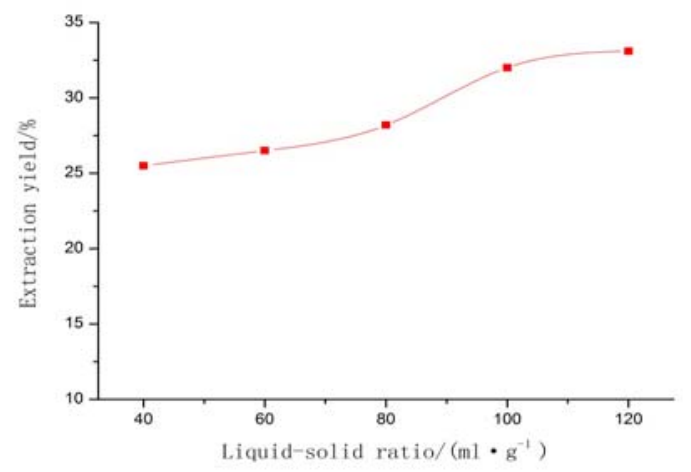

Fig.1 Liquid ratio of polysaccharide extraction rate diagram

It can be seen from Fig. 1 that when the liquid ratio of 40 , the extraction rate of $25.5 \%$, liquid ratio is 60 . Extraction rate of $26.5 \%$, liquid to solid ratio of 80 , the extraction rate of $28.2 \%$, liquid ratio is 100 , the extraction rate of $32.0 \%$, liquid to solid ratio of 120 . Extraction rate of $33.1 \%$, with the 
increase of liquid ratio, the extraction rate of polysaccharide from Gracilaria is also rising, indicating that the amount of the extraction solvent has a certain influence on the dissolution of Polysaccharide from Gracilaria, when the dissolution of the concentration does not reach the saturation solubility, the polysaccharide increases the amount of distilled water and continuous dissolution, so as to continuously improve the extraction rate effect.

\section{Effect of Ultrasonic Power on Extraction Rate of Polysaccharide from Gracilaria}

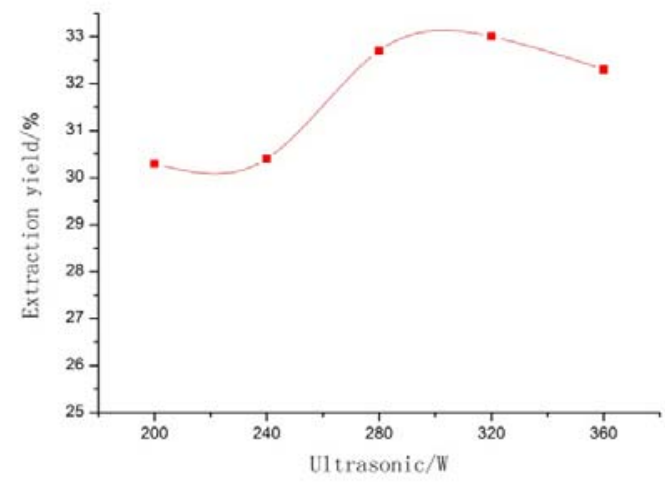

Fig.2 Ultrasonic power and polysaccharide extraction rate diagram

It can be seen from Fig. 2 that when the ultrasonic power at $200 \mathrm{~W}$, extraction rate of $30.3 \%$, when the ultrasonic power $240 \mathrm{~W}$, extraction rate of $30.4 \%$, when the ultrasonic power $280 \mathrm{~W}$, extraction rate of $32.7 \%$, ultrasonic power $320 \mathrm{~W}$, the extraction rate of $33.0 \%$, ultrasonic power $360 \mathrm{~W}$, the extraction rate of $32.2 \%$, ultrasonic power of Gracilaria polysaccharide extracted after impact with the increase of ultrasonic power and to increase the downward trend in ultrasonic power 280-320W among the best state, this may be because with the increase of ultrasonic power, mechanical grinding and ultrasonic cavitation has continued to strengthen, accelerate tissue fragmentation and promote a rapid increase in the extraction rate of polysaccharides, but to a certain extent when, beyond the asparagus dish glycan structure can withstand the strength of the structure resulting in the destruction of polysaccharide from Gracilaria or polysaccharide decomposition, thus showing an increase after the first decline in relations. Therefore, the ultrasonic extraction of polysaccharides from Gracilaria must strictly control the selection and ultrasound power, it is in the best power extraction.

\section{Ultrasonic Time on the Extraction Rate of Polysaccharide from Gracilaria}

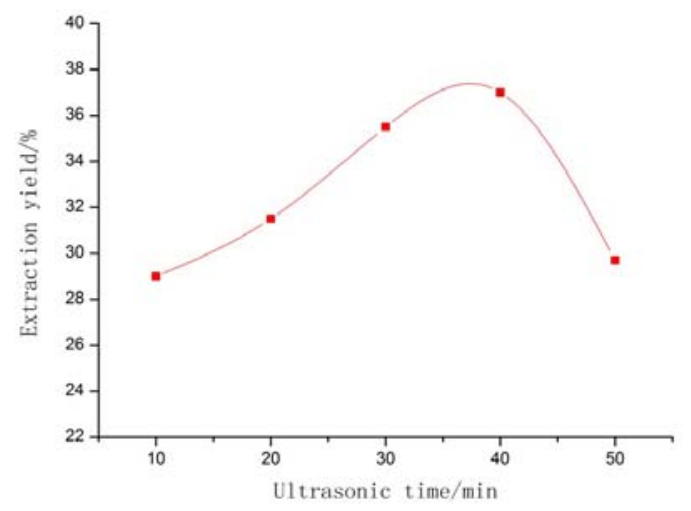

Fig.3 Ultrasound time and polysaccharide extraction rate diagram

Fig. 3 shows that when the ultrasonic time was $10 \mathrm{~min}$, the extraction rate of $29.0 \%$, ultrasonic time 
was $20 \mathrm{~min}$, the extraction rate of $31.5 \%$, ultrasonic time was $30 \mathrm{~min}$, the extraction rate of $35.3 \%$, with the increase of ultrasonic time, the dragon shall extraction rate of polysaccharides dish first and then increased and then decreased. Within a certain time, the extraction rate of polysaccharides with increasing ultrasonic time increases, which is due to the process of ultrasonic extraction of polysaccharides substantially continuously feed polysaccharide dissolution and diffusion processes. Power at the beginning, low solvent extraction polysaccharide content compared with the material content of polysaccharide, a concentration gradient is large, the polysaccharide extraction solvent eluted from the interior to the surrounding material and the rate of diffusion and large, therefore, with the increase the extraction rate of polysaccharide ultrasonic time also increases. Peak appears in $40 \mathrm{~min}$ when the extraction rate of $37.0 \%$. However, the extraction rate of polysaccharides with increasing ultrasound 40-50min time decreased significantly, ultrasonic time was 50min, the extraction rate was $29.7 \%$; this may be due to the extraction time is too long, ultrasonic waves Gracilaria polysaccharide degradation or extraction impurities out of the polysaccharide binding was removed and lost, thus showing the extraction rate. So for polysaccharide extracted from Gracilaria for full protection and polysaccharides extracted from Gracilaria, must be carefully chosen extraction time, extraction time is try to make the most appropriate stage.

\section{Effect of Temperature on the Ultrasonic Extraction Rate of Polysaccharide from Gracilaria}

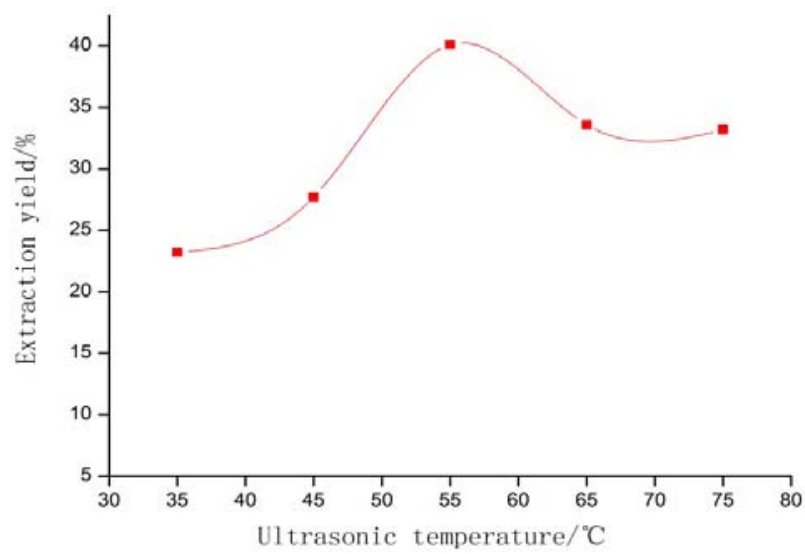

Fig.4 Ultrasonic temperature and polysaccharide extraction rate diagram

It can be seen from Fig. 4 that when the ultrasonic temperature was $35^{\circ} \mathrm{C}$, the extraction rate was $23.2 \%$; ultrasound at $45^{\circ} \mathrm{C}$, the extraction rate of $27.7 \%$; with increasing temperature of the ultrasonic extraction rate of polysaccharide from Gracilaria at first increase after decreasing trend, reaches a maximum at about $55^{\circ} \mathrm{C}$, the extraction rate of $40.1 \%$, indicating that the asparagus dish suitable ultrasonic temperature at about $55^{\circ} \mathrm{C}$. Within a certain range, the temperature of the polysaccharide helps bleeding and when the temperature exceeds the optimum conditions, the extraction rate decreased, when ultrasound at $65^{\circ} \mathrm{C}$, the extraction rate was $33.6 \%$; when the ultrasonic temperature $75^{\circ} \mathrm{C}$, extraction rate was $33.2 \%$; this may be due with increasing temperature in ultrasonic cavitation and relatively high temperature dual conditions, will polysaccharide degradation or destruction of its structure. Therefore, the need to pay attention to during the selection and extraction of polysaccharide from Gracilaria influence ultrasonic temperature to avoid damage to high temperature degradation and asparagus dish structure.

\section{Conclusion}

In this paper, by exploring ultrasonic time, ultrasonic power, extraction temperature and the ratio of liquid to solid four factors on the influence of ultrasonic extraction of polysaccharides from Gracilaria extraction rate, the following conclusions: With the increase of the ratio of liquid to solid, asparagus Polysaccharide extraction rate is also rising, ultrasonic power of Gracilaria 
polysaccharide extracted after impact with the increase of ultrasonic power and the first increase in a downward trend, with the increase of ultrasonic time, the extraction rate of polysaccharides from Gracilaria downward after the first rise the trend of. With the improvement of ultrasonic temperature, the extraction rate of polysaccharides from Gracilaria first and then increase a decreasing trend.

\section{Acknowledgements}

This research was financially supported by Guangdong Provincial Oceanic and Fishery Bureau -marine fisheries science and technology and industrial development projects (No. A201501C11 、 No. A201401C07).

\section{References}

[1]Sandra Torriani, Fausto Gardini, Maria Elisabetta Guerzoni, et al. Use of response surface methodology to evaluate some variables affecting the growth and acidification characteristics of yoghurt cultures[J].International Dairy Journal, 1996, 6(6): 625-363.

[2]Febe Francis, Abdulhameed Sabu, K. Madhavan Nampoothiri, et al. Use of response surface methodology for optimizing process parameters for the production of a-amylase by Aspergillus oryzae[J].Biochem Eng J, 2003,15(2): 107-115.

[3]S. Moyo, B.A. Gashe, E.K. Collison, S. Mpuchane. Optimising growth conditions for the pectinolytic activity of Kluyveromyces wickerhamii by using response surface methodology[J]. International Journal of Food Microbiology, 2003,85(1-2): 87-100.

[4] Chioritt i A, Liao ML, Kraft GT, et al .Cell wall polysaccharides from Australian red algae of the f amily Solieriaceae (Gigartinales, Rhodophyta):Highly methylated carrageenans from the genus Rhabdonia[J] .Bot Man, 1996 ,39(1):47-48.

[5] Brosch-Salomon S, Hof tberger M , Holzing A, et al .Ultrastructural localization of polysaccharides and N-acetyl-D-galactosamine in the secretory pathway of green algae (pesmidddiaceae)[J] .J Exp Bot ,1998, 49(319):145 .

[6] Nardella A, Chaubet F , Boisson-Vidal C, et al .Anticoagulant low molecular wt .fucans produced by radical process and ion exchangechromatography of high molecular weight fucans extracted from the brown seaweed Ascophyllum nodosum [ J] .Carbohydr Res , 1996 :289-290.

[7]Yong-guang Bi, Hai-lan Huang, Zhong Wang,Xue-wei Chen,Xue-mei Liu ,Shi-ting Deng, Hong Yu. Test Preparation Asparagus Total Polysaccharides of Scanning and Standard Curve[J]. Advances in Engineering Research,2016:1094-1098. 